\title{
Variação nas medidas corporais e desenvolvimento do trato reprodutivo de novilhas de corte recriadas para o acasalamento aos 18 meses de idade
}

\author{
Body measurements variations and development of the reproductive tract of beef heifers reared for \\ breeding at 18 months of age
}

\author{
Yuri Regis Montanholi ${ }^{I}$ Júlio Otávio Jardim Barcellos ${ }^{\mathrm{II}}$ Eduardo Castro da Costa ${ }^{\mathrm{III}}$
}

RESUMO

O objetivo deste estudo foi avaliar os efeitos do ganho de peso no período de recria, dos 13 aos 19 meses de idade, em algumas medidas de desenvolvimento corporal de novilhas de corte de reposição, da raça Hereford, mantidas em pastagem nativa. O período experimental foi compreendido entre 15/11/2002 e 23/04/2003. Os tratamentos foram três taxas de ganho de peso, em animais de 13 a 14 meses de idade, sendo: $G 600$ - 20 novilhas $(208 \pm 1,8 \mathrm{~kg})$ submetidas a um ganho diário médio (GDM) de 0,595 $\mathrm{kg} \mathrm{dia}^{-1}$; G700 - 23 novilhas $(197+1,7 \mathrm{~kg})$ submetidas a um GDM de $0,637 \mathrm{~kg} \mathrm{dia}^{-1} e$ G800 - 24 novilhas $(181 \pm 1,2 \mathrm{~kg})$ submetidas a um GDM de $0,723 \mathrm{~kg} \mathrm{dia}^{-1}$. Os GDM foram estabelecidos para que todos os animais atingissem $300 \mathrm{~kg}$ (ou $65 \%$ do peso adulto) ao início da estação de monta. Foram avaliados os efeitos dos tratamentos sobre o peso, a altura da garupa (AG), o perímetro torácico (PT), a relação peso:altura (PA) e sobre o escore de trato reprodutivo (ETR). Não foram observadas diferenças $(P>0,05)$ no incremento da AG e do PT e na PA entre os grupos experimentais. $O$ peso vivo foi altamente correlacionado $(P<0,05)$ com a $A G$, o PT e a PA ao longo de todo o período de recria. Os grupos $G 700$ e G800, submetidos a maior taxa de ganho de peso, apresentaram maiores valores $(P<0,05)$ de ETR em relação ao G600 ao início da estação de monta. A AG e a PA de novilhas de corte não foram afetadas por ganhos de peso entre 0,600 e 0,730 $\mathrm{kg} \mathrm{dia}^{-1}$ dos 13 aos 19 meses de idade. Novilhas submetidas a mais intensas taxas de crescimento (G700 e G800) demonstraram estar mais aptas a conceberem do que novilhas recriadas numa menor taxa de crescimento (G600), considerando um mesmo peso alvo ao final do perído de recria.

Palavras-chave: acasalamento no outono, altura da garupa, escore do trato reprodutivo, pastagem nativa, perímetro torácico, relação peso:altura, ganho de peso.

\begin{abstract}
This study was aimed at evaluating the effects of growth rate during the rearing phase of 13 to 19 months old replacement beef heifers Hereford on body development reared on native pasture. The experiment was conducted from 11/15/ 2002 to 04/23/2003. The treatments were the three weight gain rates: G600 - 20 heifers $(208 \pm 1.8 \mathrm{~kg})$ submitted to an average growth rate (AGR) of $0.595 \mathrm{~kg} \mathrm{day}^{-1} ; \mathrm{G} 700-23$ heifers $(197 \pm 1.7 \mathrm{~kg})$ with an AGR of $0.637 \mathrm{~kg} \mathrm{day}^{-1}$ and G800 - 24 heifers $(181 \pm 1.2 \mathrm{~kg})$ with an AGR of $0.723 \mathrm{~kg} \mathrm{day}^{-1}$. The three AGR aimed for a body weight of $300 \mathrm{~kg}$ (or $65 \%$ of the expected adult weight) at the end of the rearing phase. Treatment effects were determined on body weight, hip height $(\mathrm{HH})$, heart girth $(H G)$, weight:height ratio $(\mathrm{WH})$ and reproductive tract score (RTS). No differences $(P>0.05)$ among treatments were observed on the increment of $\mathrm{HH}, \mathrm{HG}$ and $\mathrm{WH}$ at the end of the experiment. Body weight was highly correlated to $\mathrm{HH}, \mathrm{HG}$ and WH along the whole rearing phase. The groups G700 and G800 with large growth rates showed higher RTS $(P<0.05)$ than $G 600$ at the end of the rearing phase. The $\mathrm{HH}$ and $\mathrm{WH}$ measurements taken on beef heifers are not affected by growth rates between 0.600 and $0.730 \mathrm{~kg}^{\text {day }}{ }^{-1}$ from 13 to 19 months of age. Beef heifers submitted to higher rates of growth (G700 e G800) demonstrated to be more likely to conceive than heifers reared on a lower growth rate (G600), considering the same target weight at the end of the rearing phase.
\end{abstract}

Key words: fall season breeding, heart girth, hip height, native pasture, reproductive tract scoring, weight:height ratio, weight gain.

\section{INTRODUÇÃO}

O desempenho reprodutivo é a variável de maior importância no rebanho de cria de bovinos de corte, pois, em conjunto com a produtividade,

IDepartment of Animal \& Poultry Science, University of Guelph, Ontário, Canadá. E-mail: ymontanh@uoguelph.ca. Autor para correspondência.

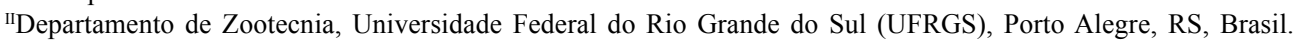

IIIFundação Estadual de Pesquisa Agropecuária (FEPAGRO), Estação Experimental de São Gabriel, RS, Brasil. 
determinam o desfrute geral do rebanho (WILTBANK, 1994). WILLHAM \& MIDDLETON (1983) demonstraram que a reprodução, em termos de valores relativos, foi 10 vezes mais importante do que a produtividade. Os índices reprodutivos de um rebanho são fortemente influenciados pelo manejo adotado nas fêmeas de reposição, pois estas repõem cerca de 10$20 \%$ das vacas anualmente (WILTBANK, 1994). Portanto, a seleção e o manejo dessas fêmeas envolvem decisões que afetam a produtividade futura de todo o rebanho (PATTERSON et al., 1992).

A máxima eficiência biológica de um sistema de produção é obtida quando as novilhas são acasaladas aos 14-15 meses de idade (SHORT et al., 1994). Entretanto, a produção desta novilha é um processo característico de sistemas intensivos de produção, com custos elevados e que correspondem a $7 \%$ dos sistemas pecuários de cria existentes no Brasil (ANUALPEC, 2006). Por outro lado, o acasalamento aos 18 meses pode ser considerado um dos possíveis elementos a constituir um sistema semi-intensivo de produção de bovinos de corte. Dessa maneira, o acasalamento de novilhas ao sobreano é uma alternativa aos sistemas de produção extensivos e intensivos, em processo de mudança no nível tecnológico adotado. $\mathrm{O}$ acasalamento aos 18 meses normalmente ocorre durante o outono, em função dos bezerros serem nascidos na maioria dos sistemas de produção de cria durante a primavera.

A categoria novilhas é considerada como de baixa produtividade, devido ao longo tempo compreendido entre o seu nascimento e o primeiro parto (BARCELLOS et al., 2003). O manejo nutricional e o peso vivo dessas fêmeas são os principais elementos que afetam a seqüência de eventos que desencadeiam a puberdade (PATTERSON et al., 1992). É sabido que novilhas de corte Hereford atingem a puberdade com cerca de $60 \%$ do peso adulto (ARIJE \& WILTBANK, 1971). Entretanto, existem os efeitos da intensidade do ganho de peso (GRASS et al., 1982) e da fase pósdesmame, quando ocorre esse ganho (CRICHTON et al., 1959). Medidas lineares como a altura e o perímetro torácico constituem outros parâmetros de desenvolvimento físico do animal que também são relacionados ao desempenho reprodutivo (JOUBERT, 1954). Destaca-se a relação do perímetro torácico e da relação peso:altura com a condição corporal (THOMPSON et al., 1983) e a alta relação entre altura, peso e idade à puberdade (VARGAS et al., 1998). O escore de trato reprodutivo desenvolvido por ANDERSEN et al. (1991) constitui uma importante ferramenta para avaliar o grau de amadurecimento do aparelho genital das fêmeas de reposição.
O objetivo deste trabalho foi verificar os efeitos da taxa de ganho de peso durante o período da recria sobre os parâmetros de desenvolvimento corporal e as suas relações com escore do trato reprodutivo de novilhas de reposição para sistemas de produção que utilizam o primeiro acasalamento aos 18 meses de idade.

\section{MATERIAL E MÉTODOS}

O experimento foi conduzido em uma propriedade particular, no município de Bagé, região fisiográfica da Campanha do Estado do Rio Grande do Sul, situada entre os paralelos $30^{\circ} 30^{\prime}$ e $31^{\circ} 56^{\prime}$ latitude sul e os meridianos $55^{\circ} 30^{\prime}$ e $54^{\circ} 30^{\prime}$ longitude. O clima da região, segundo a classificação de Köppen, é tipo subtropical, da classe $\mathrm{Cfa}$, com chuvas regularmente distribuídas durante o ano, podendo ocorrer períodos de estiagem nos meses de janeiro e fevereiro. A temperatura média do mês mais quente é $24^{\circ} \mathrm{C}$, em janeiro, e a do mês mais frio $12,5^{\circ} \mathrm{C}$, em junho (MORENO, 1961).

Os solos da região são, basicamente, representados pelo tipo Cambissolo Háplico Ta Eutrófico Vértico (EMBRAPA, 1999), que apresenta variabilidade quanto à profundidade. A pastagem nativa do local é formada por espécies de gramíneas rizomatosas e/ou estoloníferas com a presença de leguminosas, que crescem, preferencialmente, na primavera/verão. Destacam-se as presenças da gramaforquilha (Paspalum notatum), do campim-melador (Paspalum dilatatum), da grama-tapete (Axonopus compressus), do capim-rabo-de-lagarto (Coelorachis selloana), do capim-caninha (Andropogon lateralis), do pega-pega (Desmodium pratensis) e do trevo nativo (Tripholium polimorfum). Entre as espécies hibernais, destaca-se o capim-flexilha (Stipa spp.).

No dia 15/11/2002, foram selecionados os animais para alocação em cada grupo experimental, de acordo com o peso médio. Os grupos foram denominados de G800 (24 novilhas), G700 (23 novilhas) e G600 (20 novilhas), cujos pesos foram de 181 $\pm 1,2$, $197 \pm 1,7$ e $208+1,8 \mathrm{~kg}$, respectivamente. Nesta ocasião, os animais também foram identificados através de brincos numerados na orelha esquerda. Todos os animais pertenciam à raça Hereford e apresentavam idade entre 13 e 14 meses, recebendo, desde o desmame até o início do experimento, as mesmas condições de manejo. As novilhas foram submetidas ao controle de endo e ectoparasitas e vacinadas contra as enfermidades da região.

Os grupos foram mantidos em diferentes lotações (G800: 150kg ha-1; G700: 200kg ha-1 e G600: 
$\left.300 \mathrm{~kg} \mathrm{ha}^{-1}\right)$, que se constituíram nos sistemas de alimentação, com o objetivo de produzir os ganhos de peso em cada grupo, para que todas as novilhas atingissem o peso de $300 \mathrm{~kg}$ (ou $65 \%$ do peso adulto) ao final da recria (24/04/2003).

Os animais foram pesados mensalmente e as cargas ajustadas, conforme o ganho de peso no mês anterior, de forma a garantir um ganho de peso próximo ao projetado. $\mathrm{O}$ ajuste da carga foi realizado com animais reguladores. A tabela 1 apresenta os valores projetados e obtidos para peso inicial (PI), peso ao final da recria (PFR) e para o ganho diário médio (GDM). Os piquetes utilizados eram bastante homogêneos quanto à topografia e à composição botânica, com fácil acesso à água e à suplementação mineral.

Os animais foram pesados mensalmente mediante um jejum prévio de 12 horas. Nestas ocasiões, também foi realizada a mensuração do perímetro torácico (PT) e da altura da garupa (AG). No dia 23/04/ 2003 (final do período de recria), foi realizada a avaliação do escore do trato reprodutivo (ETR), através da palpação retal, conforme a metodologia proposta por ANDERSEN et al. (1991), apresentada na tabela 2.

Foram avaliadas as seguintes variáveis: peso vivo ao início e término do período de recria (PIR e PFR) e GDM na recria. Também foram analisadas medidas do PT, da AG, da relação peso:altura (PA) e os valores do ETR. Para estas últimas variáveis (PT, AG, PA e ETR), o peso inicial foi utilizado como covariável no ajuste das médias. As diferenças entre as médias ajustadas foram testadas pelo teste Tukey, segundo o modelo: $Y i j=\mu+G i+$ eij, em que: $Y i j=$ jésima resposta medida no iésimo grupo de novilhas; $\mu=$ Efeito médio; $\mathrm{Gi}=$ Efeito do ganho de peso e eij = jésimo erro associado à iésima resposta. Foram realizadas as análises de correlação de Pearson entre peso, AG, PT e PA após o ajuste para os efeitos dos tratamentos. As análises estatísticas foram feitas utilizando os aplicativos PROC GLM e PROC CORR do programa estatístico SAS, versão 9.1.3 (2004).

\section{RESULTADOS E DISCUSSÃO}

Na tabela 3, podem ser observadas as variações nas medidas do PT e da AG e os valores da PA e do ETR ao final da recria para os três grupos experimentais. $\mathrm{O}$ incremento na altura da garupa (GAG) não foi influenciado pelos tratamentos $(\mathrm{P}>0,05)$. Este resultado está de acordo com as evidências de CRICHTON et al. (1959) e de VARGAS et al. (1998). Para esses autores, a altura é menos suscetível às variações ambientais do que o peso vivo, e é atingida mais cedo na vida do animal do que o seu peso adulto. Portanto, o crescimento do esqueleto, representado pela AG (BARKER et al., 1988; COSTA, 2006), é pouco afetado pelo nível nutricional. De acordo com JOUBERT et al. (1954), a medida da AG não evidencia retardo no desenvolvimento nem mesmo em períodos de déficit nutricional. Deve-se considerar ainda que os ganhos de peso utilizados nos três grupos do presente trabalho foram relativamente altos, o que também contribuiu para não se verificarem diferenças na GAG das novilhas.

O maior ganho de peso das novilhas do G800 no período de recria (Tabela 3 ) em relação aos grupos G600 e G700 não influenciou $(P=0,12)$ na taxa de crescimento do PT (GPT). Conforme BRODY (1945), o PT pode ser utilizado como um indicador da condição nutricional dos animais. Esses efeitos foram observados mais claramente nos valores de ETR, segundo os quais as novilhas do G800 apresentavam um maior desenvolvimento do aparelho reprodutivo ao final da recria $(\mathrm{P}<0,05)$, em relação ao G600. Com relação ao G700, provavelmente a diferença no ganho de peso não foi suficiente para possibilitar um desenvolvimento do ETR de maior magnitude do que no G600. Esses resultados sugerem que ganhos de peso superiores a $0,500 \mathrm{~kg}$ dia $^{-1}$ não provocam grandes modificações ponderais no trato reprodutivo da novilha. Este resultado está de acordo com os encontrados por PEREIRA NETO \& LOBATO (1998), manejando novilhas que consumiam a ponta da pastagem ou o resíduo pós-pastejo, que encontraram valores de 3,8 e 3,2 de ETR, respectivamente. Entretanto, após a estação

Tabela 1 - Valores do peso inicial (PIR), ganho diário médio (GDM) e peso ao final da recria (PFR) projetados e observados ( \pm erro padrão) para os três grupos experimentais.

\begin{tabular}{|c|c|c|c|c|c|c|}
\hline \multirow{2}{*}{ Grupo } & \multicolumn{2}{|c|}{ PIR (kg) } & \multicolumn{2}{|c|}{ GDM $\left(\mathrm{kg} \mathrm{dia}^{-1}\right)$} & \multicolumn{2}{|c|}{ PFR (kg) } \\
\hline & projetado & observado & projetado & observado & projetado & observado \\
\hline G600 & 210 & $208^{\mathrm{a}}( \pm 1,75)$ & 0,563 & $0,595^{\mathrm{c}}( \pm 0,013)$ & 300 & $302^{\mathrm{a}}( \pm 1,99)$ \\
\hline G700 & 195 & $197^{\mathrm{b}}( \pm 1,69)$ & 0,656 & $0,637^{\mathrm{b}}( \pm 0,012)$ & 300 & $298^{\mathrm{a}}( \pm 1,92)$ \\
\hline G800 & 175 & $181^{\mathrm{c}}( \pm 1,18)$ & 0,781 & $0,723^{\mathrm{a}}( \pm 0,009)$ & 300 & $296^{\mathrm{a}}( \pm 1,34)$ \\
\hline
\end{tabular}

${ }^{\mathrm{a}, \mathrm{b}}$ Médias seguidas por letras diferentes na coluna diferem entre si pelo teste Tukey $(\mathrm{P}<0,05)$.

Ciência Rural, v.38, n.1, jan-fev, 2008. 
Tabela 2 - Descrição das categorias do escore de trato reprodutivo (ETR).

\begin{tabular}{|c|c|c|c|c|c|}
\hline \multirow[b]{2}{*}{ ETR } & \multirow[t]{2}{*}{ Cornos uterinos } & \multicolumn{4}{|c|}{ Ovários } \\
\hline & & Comprimento & Altura & Largura & Folículos \\
\hline 1 & imaturo $<20 \mathrm{~mm}$ de diâmetro, sem tônus & $15 \mathrm{~mm}$ & $10 \mathrm{~mm}$ & $08 \mathrm{~mm}$ & ausência \\
\hline 2 & 20-25mm de diâmetro, sem tônus & $18 \mathrm{~mm}$ & $12 \mathrm{~mm}$ & $10 \mathrm{~mm}$ & $8 \mathrm{~mm}$ \\
\hline 3 & 25-30mm de diâmetro, leve tônus & $22 \mathrm{~mm}$ & $15 \mathrm{~mm}$ & $10 \mathrm{~mm}$ & $8-10 \mathrm{~mm}$ \\
\hline 4 & $30 \mathrm{~mm}$ de diâmetro, bom tônus & $30 \mathrm{~mm}$ & $16 \mathrm{~mm}$ & $12 \mathrm{~mm}$ & $>10 \mathrm{~mm}$ \\
\hline 5 & $>30 \mathrm{~mm}$ de diâmetro, bom tônus, ereto & $>32 \mathrm{~mm}$ & $20 \mathrm{~mm}$ & $15 \mathrm{~mm}$ & $>10 \mathrm{~mm}$, corpo lúteo \\
\hline
\end{tabular}

Fonte: ANDERSEN et al. (1991).

de monta, foi observada uma maior taxa de prenhez nas novilhas que apresentavam maiores ETR ao final da recria neste mesmo grupo de animais (MONTANHOLI et al., 2004b), bem como uma maior concentração de partos no início da estação de parição (MONTANHOLI et al., 2004a).

A relação PA (Tabela 3) foi similar $(\mathrm{P}>0,05)$ para os três grupos, sendo os valores encontrados semelhantes $\left(2,56 \mathrm{~kg} \mathrm{~cm}^{-1}\right)$ aos recomendados por FOX et al. (1988) para novilhas aos 426 dias de idade $(2,53 \mathrm{~kg}$ $\mathrm{cm}^{-1}$ ), independentemente do tamanho do animal. Entretanto, considerando a maior influência do peso nas novilhas mais jovens sobre a sua atividade reprodutiva (WILTBANK, 1994), pode-se propor que as novilhas do presente trabalho, com cerca de 540 dias de idade, apresentaram valores de PA relativamente superiores ao preconizado por FOX et al. (1988). GRASS et al. (1982) observaram que novilhas recriadas em um alto nível de ganho $\left(0,840 \mathrm{~kg} \mathrm{dia}^{-1}\right)$ atingiram a puberdade aos 392 dias com $2,93 \mathrm{~kg} \mathrm{~cm}^{-1}$ de PAe novilhas recriadas em um nível de ganho moderado $\left(0,300 \mathrm{~kg} \mathrm{dia}^{-1}\right)$ tornaram-se púberes aos 467 dias, apresentando $2,52 \mathrm{~kg}$ $\mathrm{cm}^{-1}$ de PA.

$\mathrm{Na}$ tabela 4, são apresentadas as correlações entre peso, AG, PT e PA na recria, independentemente do grupo experimental. O peso foi correlacionado com a altura, com o PT e com a PA, nos três períodos considerados (Tabela 4). Os valores da correlação entre peso e AG observados durante a recria são inferiores aos 0,45 obtidos por THOMPSON et al. (1983). No entanto, os valoresa da correlação entre peso e PT assemelham-se aos 0,62 observados por NESAMVUNI et al. (2000), muito embora esses resultados sejam inferiores aos 0,91 obtidos por DUNN et al. (1983). A avaliação das correlações entre PT e AG com o peso evidencia que o PT é uma medida linear mais adequada para se estimar o peso do que a $\mathrm{AG}, \mathrm{o}$ que está de acordo com os resultados de THOMPSON et al. (1983) e de NELSEN et al. (1985).

A alta correlação entre peso e PA nos períodos do início e meio da recria é similar ao valor de 0,97 encontrado por THOMPSON et al. (1983). Entretanto, no período final da recria, foi observado um menor valor nessa correlação. Isso pode ser atribuído à diminuição na intensidade do crescimento esquelético das novilhas, representado pela $\mathrm{AG}$, haja vista que o incremento no peso foi de maior magnitude do que o aumento na $\mathrm{AG}$, o que repercutiu em um maior incremento na PA neste período. Esse fato ficou mais evidente quando se observou a correlação entre AG e PA. Nos dois períodos iniciais, a correlação entre essas duas variáveis não foi significativa, tornando-se negativa no período final da recria $(\mathrm{P}<0,01)$. De maneira similar, pode-se propor que a correlação observada entre AG e PT nos dois períodos iniciais, que se apresentou não-significativa no período final, também seja atribuída à diminuição do crescimento do esqueleto neste período.

A PA e o PT são relacionados à condição corporal das novilhas (NELSEN et al., 1985). No presente trabalho, foi evidenciada uma correlação positiva entre esses dois parâmetros. Entretanto, essa

Tabela 3 - Incrementos no perímetro torácico (GPT) e na altura (GAG), durante a recria, relação peso:altura (PA) e escore de trato reprodutivo (ETR) ao início do acasalamento, em relação aos grupos experimentais (médias ajustadas \pm erro padrão).

\begin{tabular}{cllll}
\hline Grupos & GPT $(\mathrm{cm})$ & GAG $(\mathrm{cm})$ & PA $\left(\mathrm{kg} \mathrm{cm}^{-1}\right)$ & ETR \\
\hline G600 & $17,31^{\mathrm{a}}( \pm 0,30)$ & $9,77^{\mathrm{a}}( \pm 0,25)$ & $2,47^{\mathrm{a}}( \pm 0,47)$ & $2,95^{\mathrm{a}}( \pm 0,43)$ \\
G700 & $16,34^{\mathrm{a}}( \pm 0,21)$ & $8,14^{\mathrm{a}}( \pm 0,17)$ & $2,56^{\mathrm{b}}( \pm 0,32)$ & $3,05^{\mathrm{ab}}( \pm 0,31)$ \\
G800 & $17,58^{\mathrm{a}}( \pm 0,18)$ & $8,59^{\mathrm{a}}( \pm 0,15)$ & $2,63^{\mathrm{b}}( \pm 0,29)$ & $4,25^{\mathrm{b}}( \pm 0,38)$ \\
\hline
\end{tabular}

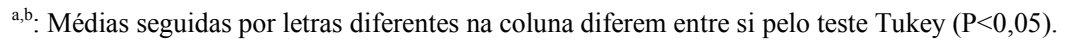

Ciência Rural, v.38, n.1, jan-fev, 2008. 
Tabela 4 - Análise global das correlações entre peso, altura da garupa (AG), perímetro torácico (PT) e relação peso:altura (PA), em três períodos da recria.

\begin{tabular}{|c|c|c|c|c|}
\hline Período & Parâmetro & $\mathrm{AG}$ & PT & PA \\
\hline \multirow{3}{*}{ Início da Recria (novembro) } & Peso & $0,331 * *$ & $0,550 * *$ & $0,897 * *$ \\
\hline & $\mathrm{AG}$ & 1 & $0,229 *$ & ns \\
\hline & PT & --- & 1 & $0,481 * *$ \\
\hline \multirow{3}{*}{ Durante a recria (fevereiro) } & Peso & $0,383 * *$ & $0,512 * *$ & $0,868 * *$ \\
\hline & $\mathrm{AG}$ & 1 & $0,224 *$ & ns \\
\hline & PT & --- & 1 & $0,432 * *$ \\
\hline \multirow{3}{*}{ Final da recria (abril) } & Peso & $0,307 * *$ & $0,552 * *$ & $0,474 * *$ \\
\hline & $\mathrm{AG}$ & 1 & ns & $-0,681 * *$ \\
\hline & PT & --- & 1 & $0,348 * *$ \\
\hline
\end{tabular}

$* \mathrm{P}<0,05 ; * * \mathrm{P}<0,01 ; \mathrm{ns} \mathrm{P}>0,05$.

correlação foi diminuindo com o avançar do período de recria, evidenciando que, no período de crescimento mais intenso, esses parâmetros estiveram mais correlacionados. O resultado da correlação entre PA e PT encontrada no presente trabalho é inferior ao valor de 0,88 observado por THOMPSON et al. (1983).

\section{CONCLUSÕES}

A taxa de ganho de peso dos 13 aos 18 meses não influenciou a altura da garupa, o perímetro torácico e a relação peso:altura de novilhas de corte de reposição Hereford. Esses dois últimos parâmetros foram mais adequados que a altura da garupa para se estimar o peso vivo de novilhas de corte.

Novilhas recriadas em taxas de ganho mais altas apresentam maiores valores de escore de trato reprodutivo, no final da recria, quando alcançam $300 \mathrm{~kg}$ de peso vivo, sugerindo melhores chances de concepção durante o acasalamento aos 18 meses de idade.

\section{AGRADECIMENTO}

Ao Conselho Nacional de Desenvolvimento Científico e Tecnológico $(\mathrm{CNPq})$, pela concessão de bolsa ao pesquisador Barcellos.

\section{REFERÊNCIAS}

ANDERSEN, K.J. et al. The use of reproductive tract scoring in beef heifers. Agri-practice, v.12, n.4, p.19-26, 1991

ANUAlPeC. Anuário da Pecuária Brasileira. São Paulo: FNP, 2006. 369p.

ARIJE, G.F.; WILTBANK, J.N. Age and weight at puberty in Hereford heifers. Journal of Animal Science, v.33, n.2, p.401-406, 1971 .
BARKER, J.F. et al. Multiple regression and principal components analysis of puberty and growth in cattle. Journal of Animal Science, v.66, n.11, p.2147-2158, 1988.

BARCELLOS, J.O.J. et al. Crescimento de fêmeas bovinas de corte aplicado aos sistemas de cria (Sistemas de produção em bovinos de corte. Porto Alegre : Departamento de Zootecnia, Universidade Federal do Rio Grande do Sul, 2003. 72p. (Publicação Ocasional, 1).

BRODY, S. Bioenergetics and growth. New York: Reinhold, 1945. 1023p.

COSTA, E.C. Crescimento pós-desmama e taxa de prenhez de novilhas de corte acasaladas aos 18 meses de idade. 2006. 154f. Tese (Doutorado em Zootecnia) Programa de Pós-graduação em Zootecnia, Faculdade de Agronomia, Universidade Federal do Rio Grande do Sul.

CRICHTON, J.A. et al. The effect of plane of nutrition during rearing on growth, production, reproduction and health of dairy cattle. I Growth to 24 months. Animal Production, v.1, n.2, p.145-162, 1959.

DUNN, T.G. et al. Body condition scores and carcass energy content in postpartum beef cows. Proceedings of the Western Section of the American Society of Animal Science, v.34 p.56, 1983.

EMBRAPA. Centro Nacional de Pesquisa de Solos Rio de Janeiro - RJ. Sistema Brasileiro de Classificação de Solos. Brasília: Embrapa Produção de Informação; Rio de Janeiro: Embrapa Solos, 1999. 412p.

FOX, D.G. et al. Adjusting nutrient requirementes of beef cattle for animal and environmental variations. Journal of Animal Science, v.66, n.5, p1475-1453, 1988.

GRASS, J.A. et al. Genotype x environmental interactions on reproductive traits of bovine females. I. Age at puberty as influenced by breed, breed of sire, dietary regimen and season. Journal of Animal Science, v.55, n.6, p.1441-1457, 1982. 
JOUBERT, D.M. The influence of winter nutritional depressions on the growth, reproduction and production of cattle. Journal of Agricultural Science, v.44, p.5-65, 1954.

MONTANHOLI, Y.R. et al. Distribuição da parição de novilhas de corte recriadas em diferentes taxas de ganho de peso dos 13 aos 18 meses de idade e acasaladas ao sobreano no outono. In: REUNIÃO ANUAL DA SOCIEDADE BRASILEIRA DE ZOOTECNIA, 41., 2004, Campo Grande. Anais... Campo Grande, MS: SBZ, 2004a. 1 CD.

MONTANHOLI, Y.R. et al. Ganho de peso na recria e desempenho reprodutivo de novilhas acasaladas ao sobreano no outono. Pesquisa Agropecuária Brasileira, v.39, n.12, p.1253-1259, 2004b.

MORENO, J.A. Clima do Rio Grande do Sul. Porto Alegre: Secretaria da Agricultura, 1961. 42p.

NELSEN, T.C. et al. Palpated and visually assigned condition scores compared with weight, height and heart girth in Hereford and crossbred cows. Journal of Animal Science, v.60, n.2, p.363-368, 1985 .

NESAMVUNI, A.E. et al. Estimation of body weight in Ngunitype cattle under communal management conditions. South African Journal of Animal Science, v.30 (suppl.1), p.9798, 2000 .

PATTERSON, D.J. et al. Management considerations in heifer development and puberty. Journal of Animal Science, v.70, n.12, p.4018-4035, 1992.
PEREIRA NETO, O.A.; LOBATO, J.F.P. Efeitos da ordem de utilização de pastagens nativas melhoradas no desenvolvimento e comportamento reprodutivo de novilhas de corte. Revista da Sociedade Brasileira de Zootecnia, v.27, n.1, p.60-65, 1998 .

SAS. SAS OnlineDoc: SAS Institute Inc. Version 9.1.3. Cary, NC, 2004. 1 CD.

SHORT, R.E. et al. Breeding heifers at one year of age: biological and economic considerations. In: FIELDS, M.J.; SAND, R.S. Factors affecting calf crop. Boca Raton: CRC, 1994. p.5568.

THOMPSON, W.R. et al. Linear measurements and visual appraisal as estimators of percentage empty body fat of beef cows. Journal of Animal Science, v.56, n.4, p.755-760, 1983.

VARGAS, C.A. et al. Estimation of genetic parameters for scrotal circumference, age at puberty in heifers, and hip height in Brahman cattle. Journal of Animal Science, v.76, n.10, p.2536-2541, 1998.

WILLHAM, R.L.; MIDDLETON, B.K. The design of creative breeding programs. In: BAKER, F.H. Beef cattle science handbook. Boulder: Westview, 1983. p.299-309.

WILTBANK, J.N. Challenges for improving calf crop. In: FIELDS, M.J.; SAND, R.S. Factors affecting calf crop. Boca Raton: CRC, 1994. p.55-68. 